\title{
A Soft Computing Approach for Osteoporosis Risk Factor Estimation
}

\author{
Dimitrios Mantzaris ${ }^{1}$, George Anastassopoulos ${ }^{2}$, Lazaros Iliadis ${ }^{3}$, \\ Konstantinos Kazakos ${ }^{4}$, and Harris Papadopoulos ${ }^{5}$ \\ ${ }^{1}$ Informatics Laboratory, Department of Nursing, \\ Technological Educational Institute of Kavala, GR-68300, Didymoteicho, Greece \\ dmantzar@med.duth.gr \\ ${ }^{2}$ Medical Informatics Laboratory, Democritus University of Thrace, GR-68100, \\ Alexandroupolis, Greece \\ anasta@med.duth.gr \\ ${ }^{3}$ Department of Forestry \& Management of the Environment and Natural Resources, \\ Democritus University of Thrace, GR-68200, Orestiada, Hellas \\ liliadis@fmenr.duth.gr \\ ${ }^{4}$ Department of Orthopedics, University Hospital of Alexandroupolis, Medical School, \\ Democritus University of Thrace, GR-68100 \\ kazakosk@yahoo.gr \\ ${ }^{5}$ Department of Computer Science and Engineering, Frederick University, \\ 7 Y. Frederickou St., Palouriotisa, Nicosia 1036, Cyprus \\ h.papadopoulos@frederick.ac.cy
}

\begin{abstract}
This research effort deals with the application of Artificial Neural Networks (ANNs) in order to help the diagnosis of cases with an orthopaedic disease, namely osteoporosis. Probabilistic Neural Networks (PNNs) and Learning Vector Quantization (LVQ) ANNs, were developed for the estimation of osteoporosis risk. PNNs and LVQ ANNs are both feed-forward networks; however they are diversified in terms of their architecture, structure and optimization approach. The obtained results of successful prognosis over pathological cases lead to the conclusion that in this case the PNNs (96.58\%) outperform LVQ $(96.03 \%)$ networks, thus they provide an effective potential soft computing technique for the evaluation of osteoporosis risk. The ANN with the best performance was used for the contribution assessment of each risk feature towards the prediction of this medical disease. Moreover, the available data underwent statistical processing using the Receiver Operating Characteristic (ROC) analysis in order to determine the most significant factors for the estimation of osteoporosis risk. The results of the PNN model are in accordance with the ROC analysis and identify age as the most significant factor.
\end{abstract}

Keywords: Artificial Neural Networks, Probabilistic Neural Networks, Learning Vector Quantization Neural Networks, ROC analysis, Osteoporosis.

\section{Introduction}

Artificial Neural Networks, Genetic Algorithms and Fuzzy Logic are modern subfields of the emerging field of Computational Intelligence and are used in order to 
solve problems without prior knowledge and symbolic representation of their rules. Methods and algorithms derived from these subfields exhibit certain advantages and drawbacks when applied to real-life problems.

The huge mass of applications, in which ANNs can be used with promising results [1] is the essential element of their growth. ANNs have been widely implemented over the last years in many scientific areas, like the industry [2], image processing [3], communications [4] and medicine [5-7].

Physicians use medical protocols in order to diagnose diseases. Medical diagnosis is the accurate decision of upon the nature of a patient's disease, the prediction of its likely evolution and the chances of recovery by a physician, based on a set of clinical and laboratorial criteria, applicable to a particular case.

ANNs are suitable for disease prognosis since there is no need to provide a diagnostic rule to identify the disease, but a set of examples that represent the variations of the disease. ANNs have been used in many medical areas successfully, such as cardiology [8], oncology [9], orthopaedics [5], urology [9], surgery [6] etc.

The determination of the suitable ANN architecture is a critical point in some applications. An ANN with a small number of neurons involves deficient training, whereas a large number of processing elements limits its generalization ability. A typical approach for the proper design of an ANN is trial and error.

This study employees best-performance Probabilistic Neural Networks (PNN) [5] and also Learning Vector Quantization (LVQ) ones, which do not implemented in similar research efforts for osteoporosis estimation in the past [5], [10-13]. Moreover, the implemented ANNs are based on a vast data set comprising of 3426 records corresponding to an equal number of patients, whereas previous studies [10-13] were based on data sets with fewer cases.

The PNN with the best performance, as well as the Receiver Operating Characteristic (ROC) analysis were used for the investigation of the essential diagnostic factors of osteoporosis.

\section{Osteoporosis and Data}

Osteoporosis is a common bone disease that leads to an increased risk of fracture. In osteoporosis, the Bone Mineral Density (BMD) is reduced; the bone microarchitecture is disrupted whereas the concentration and the variety of proteins in bones are altered. Apart from the direct physical implications of a fracture, such as pain and inconvenience, osteoporotic fractures (involving the hip or the spine) are a major cause of morbidity and mortality.

Osteoporosis is a most frequent disease for women after menopause, when it is called postmenopausal osteoporosis, but it may also be developed in men. It may occur in anyone in the presence of particular hormonal disorders and other chronic diseases or as a result of medications, specifically glucocorticoids. In this case the disease is called steroid or glucocorticoid induced osteoporosis. It is a fact that a percentage as high as $75 \%$ of the women with osteoporosis disregards this disorder.

The diagnosis of osteoporosis is based on measuring the BMD of the patient. The most popular laboratorial method for doing this is the Dual Energy X-ray Absorptiometry (DEXA) scan. This examination, applied to specific bones, is costly and based 
on radiation absorption from the patient, so it is not recommended in all orthopedic cases.

The osteoporosis data, which were used for the design of the PNN and LVQ models, were obtained from the Orthopedic Clinical Information System of Alexandroupolis' University Hospital in Greece. This information system consists of a main database (MDB) where all medical records and statistical data is kept. For secure and real-time connection to the database, the information has been distributed, designing a replication scheme of the related database MDB. Although, the data belong to different instances, they seem to be included in a single database [14].

The healthcare specialists access the medical data via a friendly and ergonomic web interface. The users of the Orthopedic Clinical Information System have remote access to the MDB either by their desktop personal computers or by Personal Digital Assistants (PDAs) wireless connected to database.

Data related to four diagnostic factors were stored for each patient, namely: age, sex, height and weight. The diagnostic factors for osteoporosis risk prediction as well as their values are presented in Table 1 and described in detail in [5].

Table 1. Osteoporosis diagnostic factors coding

\begin{tabular}{ccccc}
\hline NN inputs & 1 & 2 & 3 & 4 \\
Variables & Age & Sex & Height & Weight \\
Coding & Numeric value (years) & 1 (Female) 2 (Male) & Numeric value (cm) & Numeric value (kg) \\
\hline
\end{tabular}

The estimation of the osteoporosis risk factor was based on a T-score value, which is the patient's bone density compared to the normally expected in a healthy young adult of the specific sex. A T-score value less than or equal to -2.5 implies the development of osteoporosis, whereas a T-score greater than -2.5 is related to a normal person. These values of bone densitometry were divided into 2 classes, as presented in Table 2.

As it has already been mentioned, the present study is based on a data set comprising of 3426 records. Actually, the vast majority 3346 cases $(97.7 \%)$ are related to women and only 80 cases $(2.3 \%)$ are related to men with osteoporosis symptoms. The 1083 persons are pathological cases, as their T-score was less than or equal to -2.5 .

Table 2. T-score values coding

\begin{tabular}{cc}
\hline T-score Value & Classification \\
\hline$\leq-2.5$ & 1 \\
$>-2.5$ & 2 \\
\hline
\end{tabular}

This data set was divided into a set of 2426 records for the training of both PNNs and LVQ ANN and another set of 1000 records for the evaluation and testing of their classification efficiency. 


\section{PNNs and LVQ Models in Medical Diagnosis Prediction}

In this study, two osteoporosis risk prediction models based on a non-symbolic learning approach are presented. These proposed models use ANNs, particularly Probabilistic Neural Networks (PNNs) and Learning Vector Quantization (LVQ) ones.

PNNs are a variant of Radial Basis Function Networks and approximate Bayesian statistical techniques. The modus operandi of PNNs is familiar to the human decision making approach [8]. The patterns' classification by PNNs is based on Parzen's Probabilistic Density Function (PDF) estimator [8]. A PNN is a feed-forward neural network, consisting of two layers. The first layer, which consists of radial basis function units, computes the distances between each input vector and the training input vectors [15]. The obtained vector's values indicate the propinquity of the input vector to a training input. The second layer, which is a competitive layer, sums these contributions for each class of inputs to produce a vector of probabilities as output. These probabilities indicate the likelihood of the input vector to be classified in each of the available classes. The transfer function of the second layer detects the maximum probability and classifies the input vector to the corresponding class.

More details about the specific implementation of the used PNNs can be found in [5]. The PNN learns with exposure to training patterns with a single pass without the necessity of extended training. This feature implies that the PNN may manage large quantities of data much faster that other ANN architectures. The maximum accuracy requires the determination of a "smoothing factor", which represents the width of the calculated Gaussian curve for each probability density function.

The LVQ neural networks were first proposed by Kohonen. A LVQ network is a two-layer feed-forward network, consisting of a competitive layer and a linear layer. The first layer learns to classify the input vectors in the same way as the competitive layer of PNNs. The second layer transforms the competitive layer's classes into desired classifications defined by the designer of LVQ network. The classes of the competitive layer are called subclasses while the classes of the linear layer are called target classes [16]. Both of the competitive and linear layers have one neuron per class. The number of neurons for the hidden layer is always larger than the number of output neurons.

The LVQ method is used in training ANNs for pattern classification, where each output represents a particular class. Each class is referred by a vector of weights that sequentially, represents the centers of the classes. The training data set is used several times during the training phase in a random order. The training of LVQ ANNs is terminated when classes remain stable or a specific number of iterations has been carried out. A trained LVQ neural network is a vector comparator. When a new vector is presented to the input layer of a LVQ ANN it will be classified to a class with the closest center.

\section{Experimental Results}

The MATLAB Neural Network Toolbox was used for the construction and the assessment of the classification efficiency of the developed PNN and LVQ neural networks. 
The effectiveness as well as the user-friendly interface conduced to the selection of the specific software for the purpose of this study.

The implemented PNN and LVQ architectures, the number of neurons in the input, hidden and output layers, as well as the transfer functions used in the hidden and the output layers for each network type, are summarized in Table 3. The spread of the radial basis function, representing the width of the Gaussian curve, varied during the experimental design phase of the PNNs. The three level architecture of the optimal PNN is 4-2426-2, whereas the spread value offering the best performance was equal to 0.6 .

The three level architecture of the optimal LVQ neural network was 4-4-2. A statistical analysis over osteoporosis data set indicated that the data is grouped into four subclasses. As it was mentioned, the number of hidden neurons of an LVQ neural network equals to the number of subclasses, so the implemented LVQ has four neurons for its hidden layer.

Table 3. The optimal ANNs for the osteoporosis risk factor prediction

\begin{tabular}{rccccc}
\hline \multirow{2}{*}{ ANNs } & $\begin{array}{c}\text { Input Layer - } \\
\text { Neurons }\end{array}$ & \multicolumn{2}{c}{ Hidden Layer } & \multicolumn{2}{c}{ Output Layer } \\
\cline { 3 - 5 } & 4 & Transfer Function & Neurons & Function & Neurons \\
\hline \multirow{2}{*}{ PNN } & 4 & $\begin{array}{c}\text { Radial Basis } \\
\text { (spread = 0.6) }\end{array}$ & 2426 & $\begin{array}{c}\text { Competitive } \\
\text { (compet) }\end{array}$ & 2 \\
LVQ & 4 & Competitive & 4 & Linear & 2 \\
\hline
\end{tabular}

The obtained results from the optimal PNNs and LVQ ANNs for the osteoporosis risk prediction are summarized in Table 4. The performances of the proposed ANN models are depicted in columns two to seven. In this study, the classification efficiency of PNNs and LVQ ANNs was based on two criteria. The first criterion was the percentage (numbers in parenthesis correspond to real cases) of correct classified cases over testing (1000 cases), training (2426 cases) and overall data set (3426 cases). These percentages are recorded in columns two to four of Table 4. An important criterion for the evaluation of the ANN's generalization ability is the proper classification of the pathological cases. The implemented ANNs have to recognize patients with increased osteoporosis risk factor, so the fields five to seven store the percentage of pathological cases that have been categorized correctly, for testing, training and overall data, respectively.

Table 4. Experimental results using PNN and LVQ architectures

\begin{tabular}{ccccccc}
\hline \multirow{2}{*}{$\begin{array}{c}\text { ANN } \\
\text { model }\end{array}$} & \multicolumn{2}{c}{ Percentage of Successful Prognosis } & \multicolumn{3}{c}{$\begin{array}{c}\text { Percentage of Successful Prognosis Over } \\
\text { Pathological Situations }\end{array}$} \\
\cline { 2 - 7 } & Testing Set & Training Set & Overall Set & Testing Set & Training Set & Overall Set \\
\hline \multirow{2}{*}{ PNN } & 83.10 & 84.95 & 84.41 & 95.90 & 96.87 & 96.58 \\
& $(831)$ & $(2061)$ & $(2892)$ & $(304)$ & $(742)$ & $(1046)$ \\
\multirow{2}{*}{ LVQ } & 82.60 & 82.11 & 82.25 & 94.95 & 96.48 & 96.03 \\
& $(826)$ & $(1992)$ & $(2818)$ & $(301)$ & $(739)$ & $(1040)$ \\
\hline
\end{tabular}


It is shown clearly that PNN outperforms LVQ neural network, as the percentages of successful prognosis for overall and pathological cases for testing, training and for the entire data set are higher. Therefore, the PNN has been characterized as the optimal choice of ANN for being potentially used towards osteoporosis risk prediction.

The PNN was used for the significance estimation of the diagnostic risk factors and it was trained with 4 pruned data sets. Each of these sets used values related to 3 diagnostic factors instead of the total 4 . In other words, one different diagnostic factor was omitted in each data set. The PNN was constructed and tested with the pruned data sets, and the obtained results are recorded in Table 5. The first column presents the diagnostic factor which was not considered during the PNN's development. The 2nd to 7th columns depict the performance of PNN for each of pruned data sets. The results of the 2 nd to 4 th columns are the percentages of successful prognosis over testing (1000 cases), training (2426 cases) and overall pruned data sets (3426 cases). The 5th to 7 th columns record the percentage of successful prognosis of pathological cases for testing, training and overall pruned data sets.

Table 5. Experimental results using Pruned PNN

\begin{tabular}{ccccccc}
\hline \multirow{2}{*}{$\begin{array}{c}\text { Omitted } \\
\text { Factor }\end{array}$} & \multicolumn{2}{c}{ Percentage of Successful Prognosis } & \multicolumn{3}{c}{$\begin{array}{c}\text { Percentage of Successful Prognosis Over } \\
\text { Pathological Situations }\end{array}$} \\
\cline { 2 - 6 } & Testing Set & Training Set & Overall Set & Testing Set & Training Set & Overall Set \\
\hline Age & 82.90 & 83.02 & 82.98 & 94.95 & 96.01 & 95.75 \\
Sex & 83.10 & 84.87 & 84.35 & 95.58 & 96.87 & 96.49 \\
Height & 83.70 & 82.98 & 83.19 & 99.68 & 99.22 & 99.35 \\
Weight & 83.00 & 82.85 & 82.89 & 99.68 & 99.87 & 99.82 \\
\hline
\end{tabular}

The results of the pruned PNN were compared to the ones related to the full-sized PNN. The performance of PNN dropped dramatically in the case of the omission of the "age" feature. The PNN's effectiveness remained stable when the "sex" diagnostic factor was omitted, as the vast majority is women with osteoporosis symptoms. The absence of "height" improved the PNN's performance.

The omission of "weight" did not improve the classification efficiency of the pruned PNN according to the percentages of successful prognosis for testing, training and overall data set, however the percentages of successful prognosis over pathological situations are better than ones of the full-sized data set PNN. The results of Table 5 prove that the height and weight diagnostic factors reduce the generalization ability of PNN.

\section{ROC Analysis}

The available data set of osteoporosis records underwent statistical processing using Receiver Operating Characteristic (ROC) analysis for the determination of the contribution of each diagnostic factor to osteoporosis risk prediction [17].

The essential statistic from ROC is the Area Under Curve (AUC) for each diagnostic factor. The mathematical expressions for AUC, sensitivity and specificity are explained in [17]. The obtained results are summarized in Table 6. The 1st line presents the osteoporosis' diagnostic factors, while the AUC of ROC for each factor is recorded in the 2 nd line of the Table 6. 
As mentioned above, the AUC is the value of visual depiction of ROC. An AUC value greater that 0.5 indicates the importance of the contribution of a specific diagnostic factor. According to results of Table 6, the most important diagnostic factor is Age. The Sex's AUC is equal to 0.5 , so this diagnostic factor seems not to have important contribution for osteoporosis risk estimation. This result was expected as the used data set consists of 3346 women, while the number of men is negligible.

Table 6. Area of diagnostic factors' curves

\begin{tabular}{ccccl}
\hline Diagnostic Factor & Age & Sex & Height & Weight \\
\hline AUC & 0.646 & 0.503 & 0.560 & 0.641 \\
\hline
\end{tabular}

The results of pruned PNN and ROC analysis are convergence in terms of proposed diagnostic factors for osteoporosis risk prediction. Consequently, the Age factor is strongly recommended to be recorded for each patient.

\section{Conclusions}

Despite the fact that osteoporosis is an enormous public health problem with huge recourses required to deal with the immediate and long-term effects of fractures, no investigation has been performed on the effectiveness and weightiness of the various diagnostic factors in the clinical evaluation of patients. This paper evaluates the performance of two ANN topologies for osteoporosis risk prediction. The PNN outperformed the LVQ neural network in terms of successful prognosis of osteoporosis.

The PNN, which had the best performance, was used for further processing of the osteoporosis prediction data set. The PNN was designed, implemented and tested with pruned versions of the data set, for the evaluation of the most significant diagnostic factor towards osteoporosis risk. It was concluded that "age" is the most significant factor towards osteoporosis prediction.

Moreover, the available data were processed using the ROC analysis approach. The results of the optimal PNN were in accordance with the output of the ROC analysis.

In future work, it would be preferable to make the same experiments in an extended data set consisting of more male patients and more factors (such as smoking), which affect the presence of osteoporosis. The limited number of male data records used was an obstacle in the effort to reach generalized conclusions related to the "sex" factor. The additional diagnostic factors would contribute to an extensive study in terms of the osteoporosis disease. Also the scoring of the T-code values can be implemented by the use of fuzzy logic, offering a more comprehensive approach.

\section{References}

1. Dayhoff, J., DeLeo, J.: Artificial Neural Networks Opening the Black Box. Cancer Supplement 91, 1615-1635 (2001)

2. Iliadis, L.: An Intelligent Artificial Neural Network Evaluation System Using Fuzzy Set Hedges: Application in Wood Industry. In: The Annual IEEE International Conference on Tools with Artificial Intelligence (2007) 
3. Hisashi, A., Tsuyoshi, O., Takahashi, N., Tanaka, M.: Sigma-Delta Cellular Neural Network for 2D Modulation. Neural Networks 21, 349-357 (2008)

4. Haralambous, H., Papadopoulos, H.: 24-hour Neural Network Congestion Models for Highfrequency Broadcast Users. IEEE Transactions on Broadcasting 55, 145-154 (2009)

5. Mantzaris, D., Anastassopoulos, G., Lymperopoulos, K.: Medical Disease Prediction Using Artificial Neural Networks. In: 8th IEEE International Conference on BioInformatics and BioEngineering (2008)

6. Papadopoulos, H., Gammerman, A., Vovk, V.: Confidence Predictions for the Diagnosis of Acute Abdominal Pain. In: AIAI 2009, pp. 175-184 (2009)

7. Economou, G.-P.K., Mariatos, E., Economopoulos, N., Lymberopoulos, D., Goutis, C.: FPGA Implementation of Artificial Neural Networks: An Application on Medical Expert Systems. In: 4th Int. Conf. on Microelectronics for Neural Networks and Fuzzy Systems, pp. 287-293 (1994)

8. Orr, R.: Use of a Probabilistic Neural Network to Estimate the Risk of Mortality after Cardiac Surgery. J. Medical Decision Making 17, 178-185 (1997)

9. Anagnostou, T., Remzi, M., Djavan, B.: Artificial Neural Networks for Decision-Making in Urologic Oncology. Reviews in Urology 5, 15-21 (2003)

10. Lemineur, G., Harba, R., Kilic, N., Ucan, O., Osman, O., Benhamou, L.: Efficient Estimation of Osteoporosis Using Artificial Neural Networks. In: 33rd Annual Conf. of IEEE Industrial Electronics Society (IECON), pp. 3039-3044 (2007)

11. Chiu, J., Li, Y., Yu, F., Wang, Y.: Applying an Artificial Neural Network to Predict Osteoporosis in the Elderly. Studies in Health Technology and Informatics 124, 609-614 (2006)

12. Mohamed, E., Maiolo, C., Linder, R., Pöppl, S., De Lorenzo, A.: Artificial Neural Network Analysis: A Novel Application For Predicting Site-Specific Bone Mineral Density. Acta Diabetologica 40, 19-22 (2003)

13. Rae, S., Wang, W., Partridge, D.: Artificial Neural Networks: A Potential Role in Osteoporosis. J. of the Royal Society of Medicine 92, 119-122 (1999)

14. Anastassopoulos, G., Kolovou, L., Lymperopoulos, D.: A Spatial Distributed Approach for Electronic Medical Record Administration. In: Recent Advances in Communications and Computer Science. Electrical and Computer Engineering Series, A series of Reference Books and Textbooks, WSEAS, pp. 407-412 (2003)

15. Iliadis, L.: Intelligent Information Systems and applications in risk estimation. Stamoulis Publishing, Thessaloniki (2007)

16. Gray, R.: Vector Quantization. IEEE Acoustic, Speech, and Signal Processing Magazine 1, 4-29 (1984)

17. Streiner, D., Cairney, J.: What's Under the ROC? An Introduction to Receiver Operating Characteristics Curves. The Canadian Journal of Psychiatry 52, 121-128 (2007) 